\title{
TIPIFICACIONES EN NOTOTRICHE (MALVACEAE) PARA SUDAMÉRICA AUSTRAL
}

\author{
Ana M. Cialdella \& Nataly O'Leary
}

Instituto de Botánica Darwinion (ANCEFN-CONICET), Labardén 200, CC 22, B1642HYD San Isidro, Buenos Aires, Argentina; anacialdella@darwin.edu.ar (autor corresponsal).

\begin{abstract}
Cialdella, A. M. \& N. O'Leary. 2019. Typifications in Nototriche (Malvaceae) for Southern South America. Darwiniana, nueva serie 7(2): 325-329.

Five lectotypes and two neotypes are here designated for seven species in Nototriche, as part of the ongoing study of the genus for the South American flora: N. holosericea, N. megalorrhiza, $N$. meyeni, N. pseudoglabra, N. sajamensis, N. saltensis, and N. transandina. Notes about these typifications are also provided.
\end{abstract}

Keywords. Nototriche; Southern South America; typifications.

Resumen. Cialdella, A. M. \& N. O’Leary. 2019. Tipificaciones en Nototriche (Malvaceae) para Sudamérica austral. Darwiniana, nueva serie 7(2): 325-329.

Cinco lectotipos y dos neotipos son aquí designados para siete especies de Nototriche, como parte del estudio del género para la flora de Sudamérica: N. holosericea, N. megalorrhiza, $N$. meyeni, N. pseudoglabra, N. sajamensis, N. saltensis y N. transandina. Se brindan, además, notas sobre las tipificaciones.

Palabras clave. Nototriche; Sudamérica austral; tipificaciones.

\section{INTRODUCCIÓN}

El género Nototriche Turcz. incluye aproximadamente 130 especies, distribuidas en regiones andinas de Ecuador, Perú, Chile, Bolivia y Argentina, con su mayor diversidad en Perú, donde se han registrado unas 65 especies (Chanco \& Ulloa Ulloa, 2004: 693).

Este género incluye plantas hemicriptófitas, aparentemente acaules, provistas de un eje grueso, leñoso, subterráneo (caudex, según Hill, 1909: 201), con numerosas hojas dispuestas en roseta, cuya lámina presenta diversas formas. Las flores son gamosépalas y gamopétalas, el androceo es monadelfos, compuesto por numerosos estambres fusionados por sus filamentos, carácter típico dentro de las Malvaceae; el ovario es súpero y los estigmas son numerosos y papilosos. El fruto es un esquizocarpo y las semillas son irregularmente reniformes. De acuerdo con el criterio de Krapovickas (2003), Nototriche se distingue por la ausencia de brácteas involucrales y por la unión de las estípulas a la base del pecíolo de cada hoja, formando una especie de vaina en la cual se inserta el pedicelo floral.

Nototriche fue originalmente descripto en 1863 por Turczaninow, en base a cuatro especímenes coleccionados por d'Orbigny en Bolivia, 
considerados por este autor como cuatro especies distintas. Los aportes más significativos para el conocimiento del este género fueron realizados por Hill (1906, 1909), quien describió ca. 60 nuevas especies, propuso combinaciones, elaboró una clave para la identificación de 62 especies y aportó fotografías y dibujos de los caracteres con valor taxonómico. Estos estudios fueron complementados con numerosas contribuciones posteriores del mismo autor (Hill, 1928, 1932, 1933; Burtt \& Hill, 1948) y de otros autores (Krapovickas 1950, 1951, 1953, 1957, 1973, 1988, 1999, 2003; Fryxell \& Krapovickas, 1990; Chanco, 1992).

Como parte del proyecto "Flora Argentina" [Zuloaga \& Anton (dirs.), inéd.], se revisó gran parte del material tipo de los nombres relacionados con Nototriche citados para la región en estudio. En varios casos se comprobó la necesidad de realizar lectotipificaciones o neotipificaciones, parte de las cuales fueron recientemente publicadas (Cialdella \& O'Leary, 2019).

La presente contribución completa la tipificación de los taxones de Nototriche para la región; en este caso se tratan los nombres que actualmente se consideran en sinonimia.

\section{MATERIALES Y MÉTODOS}

Las colecciones originales se consultaron en sitios de internet como JSTOR Global Plants (http://plants.jstor.org), o las páginas de los herbarios $(\mathrm{G}$, disponible en http://www.ville ge.ch/musinfo/bd/cjb/chg/; P, disponible en https://science.mnhn.fr/all/search), o bien gracias a la comunicación personal con curadores de herbario. Los materiales depositados en SI fueron estudiados en forma directa. Los especímenes se indican con el acrónimo del herbario en el que se encuentran depositados (Thiers, 2019+), seguido del código de barras, cuando lo poseen. Los nombres estudiados se presentan el orden alfabético. Para cada caso, se indica al final el nombre aceptado o de uso correcto, siguiendo el criterio de Krapovickas (2008). Los artículos del Código Internacional de Nomenclatura citados en el trabajo corresponden a la última versión del mismo ("Shenzhen Code", Turland et al. 2018).

\section{RESULTADOS}

Nototriche holoserica A. W. Hill, Bull. Misc. Inform. Kew 1927: 248. 1927. TIPO (lectotipo, aquí designado): CHILE. Región III Atacama, Vallenar, Cordillera Laguna Chica, 4200 m s.m., 1-II-1924, E. Werdermann 264 (K 000994168 !; isolectotipos CAS 0214972!, E 00285253!, G 00353284!, S-R-11234!, UC 238433!; SI47204!).

Observaciones. En el protólogo Hill menciona tres colecciones distintas de Chile: Werdermann 264, Johnston 5944 y Johnston 6046 (sintipos), sin mencionar el herbario en el que dichos materiales se encuentran. Se han podido analizar imágenes de la colección Werderman 264, con duplicados en diversos herbarios, así como también de Johnston 6046 (GH 00052961!, US 00603802 !, K 0009941701 !) y de Johnston 5944 (K 000994169!). Si bien todos estos especímenes son adecuados y representativos de la especie, el ejemplar depositado en $\mathrm{K}$ (K 000994168 !), que corresponde a la colección Werdermann 264, es aquí designado lectotipo de $N$. holoserica, ya que fue estudiado por Hill (posee etiquetas que lo certifican) y es la colección mejor representada en diversos herbarios.

Nombre aceptado: Nototriche compacta (A. Gray) A. W. Hill.

Nototriche megalorrhiza (Phil.) A. W. Hill, Bot. Jahrb. Syst. 37(5): 579. 1906. Sida megalorrhiza Phil., Fl. Atacam.: 11. 1860. Malvastrum megalorrhizum (Phil.) Baker f., J. Bot. 29: 363. 1891. TIPO (lectotipo, aquí designado): CHILE. Región XV Arica y Parinacota, Desierto de Atacama, Alto de Puquios, II-1854, R. A. Philippi s.n. (SGO 000003204!).

Observaciones. En el protólogo Philippi cita: "In cacumine, Alto de Puquios, Deserti Atacama, Februario $1854 P h$.". En el herbario del Museo de Santiago (SGO) se localizó un ejemplar (SGO 000003204) compuesto por dos plantas completas y algunos fragmentos, cuya etiqueta contiene datos que coinciden con el protólogo. 
Sin embargo, este espécimen parece estar compuesto por una mezcla de colecciones, ya que se observa otra etiqueta cuyos datos indican: "Sida macrorrhiza Phil., Cienaga redonda, prov. Atacama, S. Roman 1884", este último binomio no publicado. De acuerdo con el Art. 9.14 del CIN, el nombre de un taxón debe permanecer asociado a la parte (planta) que se corresponde mejor con la descripción original o diagnosis. En la descripción de Sida megalorrhiza Philippi menciona que la planta presenta una gruesa y conspicua raíz ("radice crassa, magna") y que las hojas tienen pecíolos de aproximadamente $13 \mathrm{~mm}$ de largo ("petiolum 7 lin. longum"); ambos caracteres pueden observarse en la planta completa que se encuentra montada en la esquina inferior izquierda del pliego, cerca del sello del herbario de Santiago (SGO 051954). Por esta razón, esta planta es aquí designada lectotipo de Sida megalorrhiza. La otra planta debe ser separada en un ejemplar distinto.

Nombre aceptado: Nototriche compacta (A. Gray) A. W. Hill.

Nototriche meyeni Ulbr., Bot. Jahrb. Syst. 42(1): 120. 1908. TIPO (neotipo, aquí designado): PERU/CHILE/BOLIVIA: In mont. Sajama vel Tacora, 4500-4800 m, M. A. Stübel 108 (K 000528256 !).

Observaciones. H. Solms-Laubach, botánico alemán que estudió en Berlín (Stafleu \& Cowan 1985), utilizó el nombre Malvastrum meyeni (1907: 126), pero sin describir un nuevo taxón (nom. nud.), para referirse a una planta coleccionada por Meyen en Abril de 1831 ("Altos de Toledo, $15500 \mathrm{ft} . ")$, guardada en el herbario de Berlín (B). Dicho autor también menciona que encontró otro ejemplar atribuible a este taxón en el herbario de Kew (K), con una etiqueta cuyos datos son: "Andes S. Chili, Lobb", y agrega que esta localidad resulta dudosa. Una año después, Ulbrich describió formalmente este taxón como Nototriche meyeni, basándose en la misma planta estudiada por Solms-Laubach, coleccionada por Meyen y guardada en B. Lamentablemente, el ejemplar de B no pudo ser localizado, muy probablemente fue destruido (R. Vogt, com. pers.).
Por lo tanto, dado que no existe material original, se designó un neotipo (Art. 9.8 del CIN). Este neotipo en $\mathrm{K}$ ( $\mathrm{K}$ 000528256) es un fragmento de otro depositado en $\mathrm{B}$, que fue identificado y citado por Hill (1909: 239) como Nototriche meyeni; la designación de este neotipo sigue, entonces, el concepto de Hill, quien ha investigado profundamente este género.

Nombre aceptado: Nototriche borussica (Meyen) A. W. Hill.

Nototriche pseudoglabra A. W. Hill, Bot. Jahrb. Syst. 37: 586. 1906. TIPO (lectotipo, aquí designado): BOLIVIA. Austro-Bolivia, Puna Patanca, 3800 m, K. Fiebrig 3186 A (K 000528298!; isolectotipos CTES 0013651!, E 00285252!, F 0062973F!, GH 00052974!, GH 000528298!, GOET 007753!, L 601750!, M 0210331!, P 02285310!, S-R-11240!, U 0003863!, US 1098036!]).

Observaciones. Luego de una detallada diagnosis, Hill cita: "Hab. Austro-Bolivia: Puna Patanca $3800 \mathrm{~m}$ (Fiebrig n. 3186A)", sin indicar el herbario en el que este material está depositado. Se han podido estudiar varias imágenes digitales de esta colección, así como también una fotografía ( $\mathrm{F}$ neg. 9359!) del duplicado depositado en el herbario de Berlín (B), luego destruido. Si bien la mayoría de los duplicados son adecuados para representar al taxón, se ha designado como lectotipo al ejemplar guardado en K (K 000528298!), el que seguramente ha sido estudiado por Hill, ya que cuenta con un dibujo a lápiz de una hoja, que fue publicado luego por dicho autor (Hill 1909: fig. 29, 5).

Nombre aceptado: Nototriche anthemidifolia (J. Rémy) A.W. Hill.

Nototriche sajamensis (Hieron.) A. W. Hill, Bot. Jahrb. Syst. 37: 579. 1906. Malvastrum sajamense Hieron., Bot. Jahrb. Syst. 21: 319. 1895. TIPO (neotipo, aquí designado): Ilustración: $M$. A. Stübel $5 i$, pl. 28, fig. 14!, en A. W. Hill, Trans. Linn. Soc. Lond., Botany $2^{\text {nd }}$. Series 7(12). 1909. 
Observaciones. En el protólogo de Malvastrum sajamense, Hieronymus indica: "Peruvia et Bolivia: crescit in montibus Tacora et Sajama alt. s. m. 4500-4800 m, mense Octobri florens (col. Peruv. N. 108; coll. Boliv. N. 5)". Hill (1909: 243) brinda una detallada explicación sobre estos materiales, coleccionados por Stübel y guardados en el herbario del Museo de Berlín (B), lamentablemente destruidos (R. Vogt, com. pers.). Por lo tanto, dado que no existe material original, se designó un neotipo (Art. 9.8 del CIN); para ello se seleccionó una ilustración publicada por Hill (1909: 243, pl. 28, fig. 14), que muestra un detalle de una hoja, dibujada a partir del ejemplar Stübel 5i (B), material citado por Hieronymus en el protólogo. Cabe agregar que en el género Nototriche los dibujos de detalles presentes en un ejemplar son muy importantes; en este caso en particular, el criterio de Hill y su análisis es indispensable.

Nombre aceptado: Nototriche alternata A.W. Hill

Nototriche saltensis A. W. Hill, Bot. Jahrb. Syst. 37: 587. 1906. TIPO (lectotipo, aquí designado): ARGENTINA. Salta. Nevado del Castillo, G. Hieronymus \& G. Niederlein 41 [error a ser corregido a G. Hieronymus \& $P$. G. Lorentz 41] (K 000528245!; isolectotipos CORD 00003468!, CTES 0013652!, F 62975F!).

Observaciones. En el protólogo de Nototriche saltensis, Hill menciona: "Argentina: Prov. Salta, Nevado del Castillo (Hieronymus et Niederlein n. 41)", sin indicar el herbario en el que este material se encuentra depositado. Se ha localizado un espécimen en el herbario de Kew (K 000528245!) bajo "Hieronymus; Niederlein 41 ", cuya etiqueta dice: "presented by Hill 269-1909", el nombre de la especie manuscrito por Hill y dibujos que fueron luego publicados por este mismo autor (Hill, 1909: fig. 29, 7-8). Este ejemplar claramente fue estudiado por Hill y muy probablemente es un fragmento del espécimen depositado en el Herbario del Museo de Berlín (B), actualmente destruido (foto F neg. 9363!).
Por estas razones, este ejemplar de $\mathrm{K}$ es designado aquí como lectotipo de Nototriche saltensis.

Los ejemplares localizados en CORD, CTES y F, si bien mencionan Hieronymus et Lorentz (o $H$. et $L$.) 41, son duplicados de la misma colección. Muy probablemente Hill haya transcripto erróneamente Hieronymous et Niederlein en el protólogo, en vez de Hieronymus et Lorentz. Hieronymous y Niederlein nunca pudieron coleccionar plantas juntos en 1873, de acuerdo con datos de Stafleu \& Cowan (1981), donde se afirma que G. Niederlein coleccionó en América del sur entre 1879 y 1886. Por esta razón, puede suponerse que los coleccionistas fueron Hieronymus y Lorentz, tal como indican las etiquetas de los ejemplares de los herbarios CORD y CTES.

Nombre aceptado: Nototriche anthemidifolia (J. Rémy) A.W. Hill

Nototriche transandina A.W. Hill, Trans. Linn. Soc. London, Bot. $2^{\text {nd }}$ Series: Botany 7(12): 226. 1909. TIPO (lectotipo, aquí designado): ARGENTINA. Prov. Mendoza. "St. Rosa de los Andes to Uspallata Pass, 3650 m, "Challenger" Expedition, H. N. Moseley s.n. (K 000528253!).

Observaciones. En el protólogo de esta especie se cita: "Prov. Mendoza, St. Rosa de los Andes to Uspallata Pass, 3650 m, Moseley, 'Chalenger Expedition'; Upper valleys, Aconcagua, P. H. Gosse, Fitzgerald Expedition (Herb. Kew)". De este modo, Hill indica haber estudiado dos colecciones (sintipos), ambas depositadas en el herbario de Kew (K 000528253 ! y K 000528254 ! respectivamente), cada una con una etiqueta cuyos datos coinciden con los del protólogo. El espécimen coleccionado por Moseley (K 000528253!) es más completo y representativo de la especie, por ello es aquí designado lectotipo de N. transandina.

Nombre aceptado: Nototriche compacta (A. Gray) A.W. Hill 


\section{BIBLIOGRAFÍA}

Burtt, B. L. \& A. W. Hill. 1948. New species of Nototriche. Kew Bulletin 3(1): 125-137.

Chanco, M. 1992. Dos nuevas especies de Nototriche (Malvaceae) del Perú. Novon 2 (1): 12-15.

Chanco, M. \& C. Ulloa Ulloa. 2004. Las especies de Nototriche (Malvaceae) de Ecuador. Sida, Contributions to Botany 21 (2): 693-703.

Cialdella, A. M. \& N. O’Leary. 2019. Typification and nomenclatural clarifications of names related to the genus Nototriche (Malvaceae). Phytotaxa 411 (3): 205-214. DOI: 10.11646/phytotaxa.411.3.4

Fryxell, P. A. \& A. Krapovickas. 1990. The Malvaceae published by Turczaninow. Contributions from the University of Michigan Herbarium 17: 173-182.

Hill, A. W. 1906. Nototriche (Malvaceae). En I. Urban (ed.), Plantae novae andinae imprimis Weberbauerianae II. Botanische Jahrbücher fur Systematik, Pflanzengeschichte und Pflanzengeographie 37: 575-587.

Hill, A. W. 1909. Revision of the genus Nototriche Turcz. Transactions of the Linnean Society of London, Botany $2^{\text {nd }}$. Series 7(12): 201-266.

Hill, A. W. 1928. New Species of Nototriche from Chile with Notes on Malvastrum. Bulletin of Miscellaneous Information (Royal Botanic Gardens, Kew) 1928(1): $17-21$.

Hill, A. W. 1932. New Species of Nototriche from Bolivia. Bulletin of Miscellaneous Information (Royal Botanic Gardens, Kew) 1932(2): 77-80.

Hill, A. W. 1933. New Species of Nototriche and Malvastrum from the Argentina. Bulletin of Miscellaneous Information (Royal Botanic Gardens, Kew) 1933(10): 485-487.

Krapovickas, A. 1950. Una nueva especie del género Nototriche (Malvaceae). Boletín de la Sociedad Argentina de Botánica 3: 170-173.

Krapovickas, A. 1951. Notas citotaxonómicas en Nototriche (Malvaceae). I. Boletín de la Sociedad Argentina de Botánica 4: 107-116.

Krapovickas, A. 1953. Notas citotaxonómicas sobre Nototriche (Malvaceae). II. Boletín de la Sociedad Argentina de Botánica 5: 51-74.

Krapovickas, A. 1957. Sobre las especies polígamas de Nototriche (Malvaceae). Lilloa 28: 269-277.
Krapovickas, A. 1973. Las especies de Nototriche publicadas por Cavanilles en "Icones et descriptiones plantarum". Boletín de la Sociedad Argentina de Botánica 15 (2-3): 285-286.

Krapovickas, A. 1988. Malvaceae. En M. N. Correa (ed.), Flora Patagónica. Colección Científica, Instituto Nacional de Tecnología Agropecuaria 8(5): 126-153.

Krapovickas, A. 1999. Malvaceae, en F.O. Zuloaga \& O. Morrone (eds.), Catálogo de las plantas vasculares de la Repúbica Argentina.II. Monographs in Systematic Botany from the Missouri Botanical Garden 74: 813-844.

Krapovickas, A. 2003. Malvaceae, en R. Kiesling (ed.), Flora de San Juan, República Argentina II: 105-135. Vazquez Mazzini, Buenos Aires.

Krapovickas, A. 2008. Malvaceae. En F. O. Zuloaga, O. Morrone \& M. J. Belgrano (eds.). Catálogo de las plantas vasculares del Cono Sur (Argentina, Sur de Brasil, Chile, Paraguay y Uruguay). Monographs in systematic botany from the Missouri Botanical Garden 107(3): 2463-2520.

Rodríguez, R., C. Marticorena, D. Alarcón, C. Baeza, L. Cavieres, V.L. Finot, N. Fuentes, A. Kiessling, M. Mihoc, A. Pauchard, E. Ruiz, P. Sanchez, \& A. Marticorena. 2018. Catálogo de las plantas vasculares de Chile. Gayana, Botánica 75 (1): 1-430. DOI: $10.4067 /$ S0717-66432018000100001

Solms-Laubach, H. 1907. Ueber eine Kleine Suite hochandiner Pflanzen aus Bolivien, die Prof. Steinmann von seiner Reise im jahre 1903 mitgebracht hat von H. Grafen zu SolmsLaubach. Botanische Zeitung 65: 119-138.

Stafleu, F.A. \& R.S. Cowan. 1981. Taxonomic literature. Ed. 2.Vol. 3.Bohn, Scheltema \& Holkema, Utrecht.980 pp.

Stafleu, F.A. \& R.S. Cowan. 1985. Taxonomic literature. Ed. 2. Vol. 5. Bohn, Scheltema \& Holkema, Utrecht. 1066 pp.

Thiers, B. [permanentemente actualizado, consulta 2019] Index herbariorum: a global directory of public herbaria and associated staff. New york: New york Botanical Garden's. Virtual Herbarium, http://sweetgum.nybg.org/ih

Turland, N.J., Wiersema, J.H., Barrie, F.R., Greuter, W., Hawksworth, D.L., Herendeen, P.S., Knapp, S., Kusber, W.-H., Li, D.-Z., Marhold, K., May, T.W., McNeill, J., Monro, A.M., Prado, J., Price, M.J. \& Smith, G.F. (Eds.) (2018) International Code of Nomenclature for algae, fungi, and plants (Shenzhen Code) adopted by the Nineteenth International Botanical Congress Shenzhen, China, July 2017. Regnum Vegetabile 159. Glashütten: Koeltz Botanical Books. DOI: 10.12705/Code.2018 